\title{
Reversed Z-effect and Z-effect phenomena in femoral neck fracture treated with a dynamic locking plate fixation: Case report
}

\author{
Radoslav Morochovič, MD, PhD ${ }^{1,2}$ (D) Miloš Kňazovický, MD ${ }^{2,3}$ (D) Martin Paulo, MD²(D, \\ Katarína Takáčová, MD² ${ }^{2}$, Rastislav Burda, MD, $\mathrm{PhD}^{1,2}$ (D) \\ ${ }^{1}$ Department of Trauma Surgery, Faculty of Medicine P.J. Šafárik University and University Hospital of L. Pasteur, Košice, Slovak Republic \\ ${ }^{2}$ Department of Trauma Surgery, University Hospital of L. Pasteur, Košice, Slovak Republic \\ ${ }^{3}$ Department of Trauma Surgery, Railway Hospital and Healthcare Center, Košice, Slovak Republic
}

Femoral head preserving surgery for femoral neck fracture (FNF) is under continuous discussion and research. ${ }^{[1,2]}$ Some internal fixation systems rely on no or minimal sliding, but most implants (e.g. parallel cancellous screws, sliding hip screw) consider sliding as an inevitable part of the healing process, during which controlled impaction across the fracture line allows bony contact between the proximal and distal fragments. ${ }^{[1,3]}$ A dynamic locking plate (DLP) is an angle-fixed construct, rotationally stable and allowing telescoping on up to four parallel screws named telescrews. ${ }^{[4]}$ The telescrew consists of a barrel which is locked to the side plate and a sliding part (lag screw) which is capable of

Received: June 09, 2020

Accepted: July 23, 2020

Published online: September 11, 2020

Correspondence: Radoslav Morochovič, MD, PhD. Department of Trauma Surgery, Faculty of Medicine P.J. Šafárik University and University Hospital of L. Pasteur, Rastislavova 43, 04190 Košice, Slovak Republic.

E-mail: rrrr@email.cz

Doi: $10.5606 /$ ehc. 2021.77186

Citation: Morochovič R, Kňazovický M, Paulo M, Takáčová K, Burda R. Reversed Z-effect and Z-effect phenomena in femoral neck fracture treated with a dynamic locking plate fixation: Case report. Jt Dis Relat Surg 2021;32(1):249-252.

(C2021 All right reserved by the Turkish Joint Diseases Foundation

This is an open access article under the terms of the Creative Commons Attribution-NonCommercial License, which permits use, distribution and reproduction in any medium, provided the original work is properly cited and is not used for commercial purposes (http://creativecommons.org/licenses/by-nc/4.0/).

\section{ABSTRACT}

The aim of this case report is to present an unusual mechanism of failure of a dynamic locking plate (DLP), previously reported in dual lag screw proximal femoral nails. A 78-year-old female patient experienced a displaced right femoral neck fracture (FNF) which was managed with DLP. At the postoperative sixth-week appointment, her hip radiographs showed that two of three telescoping screws had slid within the telescrew barrel, and one screw was fully extended and cutting through the femoral head. At the third-month checkup, radiographs revealed an unhealed FNF and $12 \mathrm{~mm}$ sliding on all three telescoping screws. At the final $11^{\text {th }}$-month follow-up, two screws were completely collapsed and the previously nonpenetrating screw was partially extended and cutting through the femoral head. Femoral neck shortening and femoral neck nonunion were noticed as well. In conclusion, the postoperative course of the case supports the hypothesis of a specific pattern of DLP failure resembling reversed Z- and Z-effects found in dual lag screw proximal femoral nails.

Keywords: Dynamic locking plate, failure pattern, femoral neck fracture, femoral neck shortening, nonunion, Z-effect.

sliding only within its barrel, so preventing the lag screw from backing out. Moreover, the lag screw cannot be detached from its barrel, a feature which should prevent its medial migration through femoral head. ${ }^{[4]}$ Avascular femoral head necrosis and fracture nonunion are considered typical surgical complications after internal fixation of displaced FNF in geriatric patients. ${ }^{[4,5]}$ Yin et al. ${ }^{[6]}$ published an analysis of the relevant literature and concluded that using DLP reduces nonunion, revision and replacement rates in treating FNF, but does not affect the cut-out rate, which, according to Biber et al., ${ }^{[7]}$ occurred in $9.6 \%$ of their DLP cases. In general, screw cut-out usually develops after the femoral 
head displaces into varus during healing, or a lag screw is located in the peripheral part of the femoral head. ${ }^{[8-10]}$ Additionally, screw cut-through can occur if the sliding mechanism malfunctions or when extensive femoral neck shortening depletes screw sliding capacity. ${ }^{[10,11]}$ Specifically in trochanteric fractures, cut-through can be seen when treated with two lag screw proximal femoral nails (PFNs) in which the Z-effect occurs. ${ }^{[12]}$ The aim of this case report is to document an unusual failure mode of a DLP with screw cut-through which resembles the Z-effect and the reversed Z-effect described in dual lag screw PFNs.

\section{CASE REPORT}

A 78-year-old female patient presented with osteoporotic (Singh III/IV) displaced right FNF after a fall at home (Figure 1a, b). ${ }^{[13]}$ The patient was on medication for diabetes mellitus type 2 and arterial hypertension, but had no history of previous injury to her right hip and walked with a cane. Following preoperative medical evaluation, the FNF was stabilized within 12 hours from admission using DLP (Targon ${ }^{\circledR}$ Femoral Neck; Aesculap ${ }^{\circledR}$ B. Braun, Tuttlingen, Germany) according to the manufacturer's instructions. There were no issues in the course of the surgery. Immediate postoperative plain radiographs of the hip showed anatomical fracture reduction, with three subchondrally placed and not fully extended telescoping screws (Figure 1c, d). Her postoperative course was uneventful and the patient was able to ambulate with a walker at discharge. Hip radiographs at discharge revealed only a minimal $(3 \mathrm{~mm})$ amount of sliding on all telescrews, with no change in telescrew position within the femoral head (Figure 1e, f). At the follow-up appointment at sixth postoperative week, the patient ambulated with a walker, felt occasional pain in the right groin and exhibited reduced range of rotational motion in that hip. Hip radiographs showed that two of the three telescoping screws had slid $6 \mathrm{~mm}$ within the telescrew barrel, but the inferior posterior telescoping screw had become fully extended and was cutting through the femoral head (Figure 2a, b). The patient was offered revision surgery for the protrusion of the screw in her hip, but she declined. At the third-month appointment, the patient ambulated with a walker, and the pain in her right groin had alleviated. Hip radiographs revealed not-healed FNF and equal $12 \mathrm{~mm}$ sliding on all three telescoping screws (Figure 2c, d). At the final $11^{\text {th }}$-month follow-up, femoral neck shortening and femoral neck nonunion were noticed (Figure 3a, b). The inferior screws were completely collapsed and the proximal screw was partially extended and cutting through the femoral head (Figure 3c, d). As the patient felt well, she opted not to have surgery for femoral neck nonunion. A written informed consent was obtained from the patient.
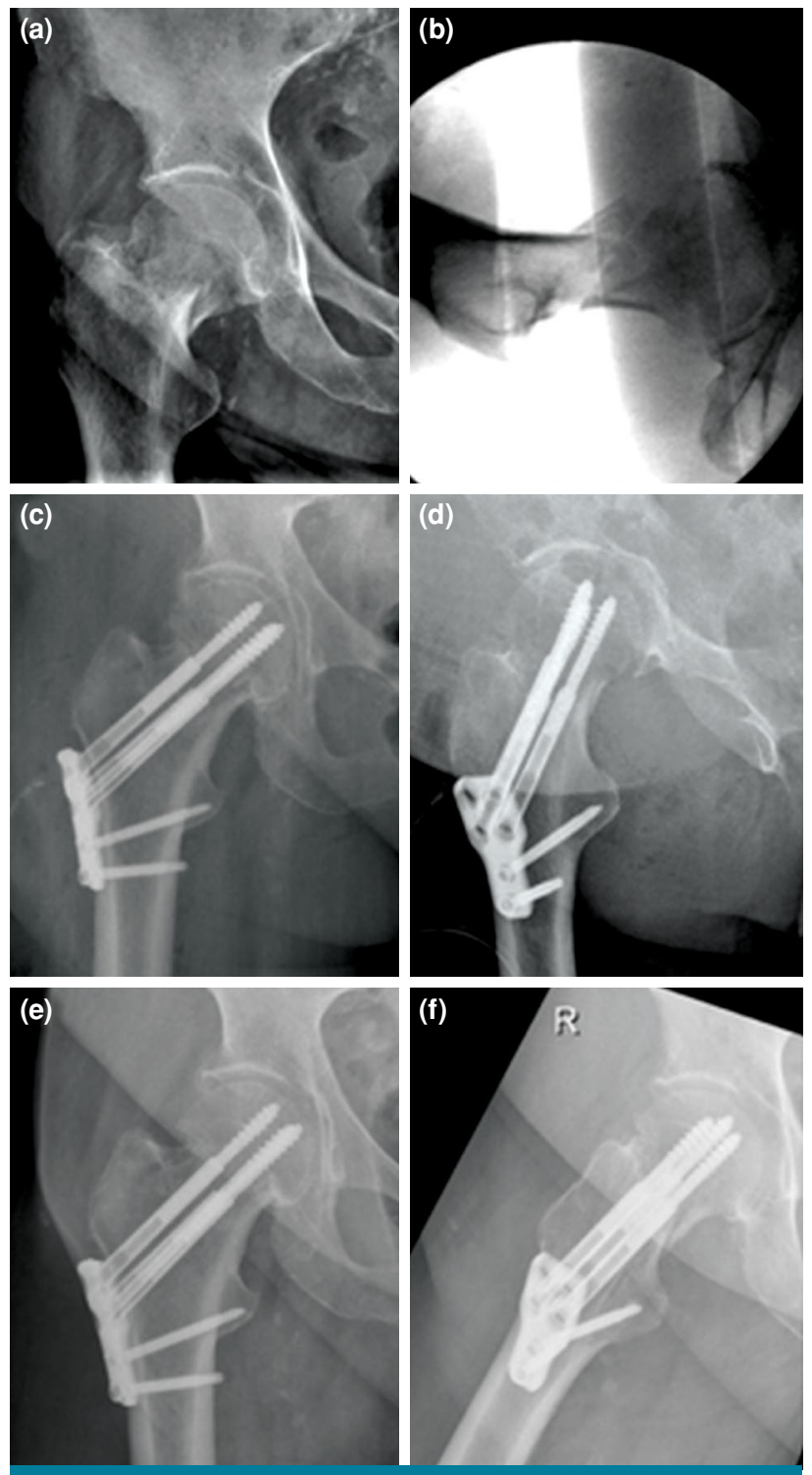

FIGURE 1. Hip radiograph of 78-year-old female patient.

(a) Radiograph shows displaced femoral neck fracture.

(b) Intraoperative hip radiograph shows medial femoral neck fragmentation. (c) Radiographs showing anatomically reduced fracture in anteroposterior and (d) lateral views. Screws are anchored subchondrally with inferior screw extension $18 \mathrm{~mm}$ and superior screw extension $12 \mathrm{~mm}$. $(e, f)$ Three millimeters of collapse in all telescoping screws at second postoperative week. Radiograph measurements were performed on a computer using Digimizer ${ }^{\circledR}$ image analysis software (version 4.6.1, MedCalc ${ }^{\circledR}$ software Ltd., Ostend, Belgium) after calibration. 

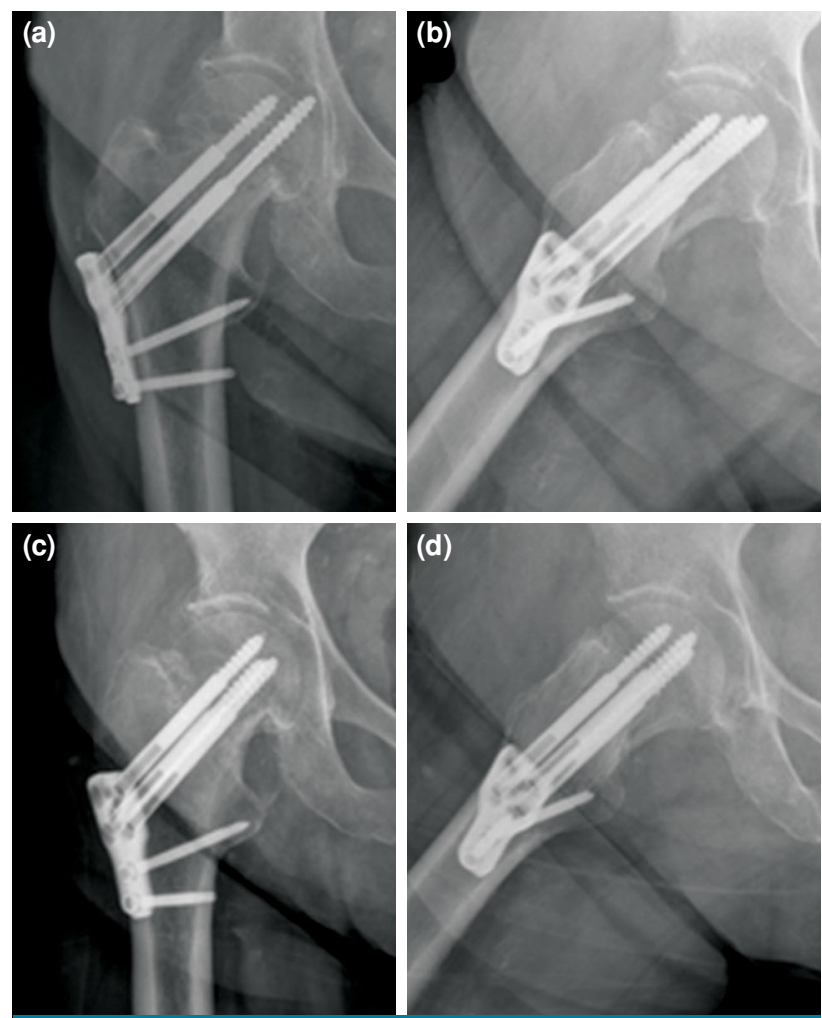

FIGURE 2. (a, b) Hip radiographs obtained at sixth postoperative week show two out of three telescoping screws sliding $6 \mathrm{~mm}$ since postoperative radiograph. Inferior posterior telescoping screw is fully extended and cutting through femoral head. (c, d) After three months, hip radiographs show $12 \mathrm{~mm}$ sliding in all three telescoping screws.

\section{DISCUSSION}

Only a few cases of cut-through in the femoral head with penetration of the hip joint using a DLP for FNF treatment have been published and reported in detail. ${ }^{[10,11]}$ Körver et al. ${ }^{[1]}$ described two such cases. In the first case, one femoral head perforating telescrew was completely collapsed and the other screws were telescoped only in part. Complete sliding of the lag screw made this telescrew rigid, and femoral neck shortening caused the screw to penetrate the hip joint. In the second case, they found two completely collapsed telescrews which were not perforating the femoral head, and a penetrating telescrew which was still minimally extended. ${ }^{[11]}$ In a case reported by Eschler et al., ${ }^{[10]}$ cut-through with hip joint penetration was seen in a telescrew which was fully extended during surgery and stayed in full extension during healing, while the other telescrews, non-penetrating ones, were already completely collapsed. The latter two cases share the same feature. The penetrating telescrews were still partially or fully extended. A plausible explanation for these failures may be loss of
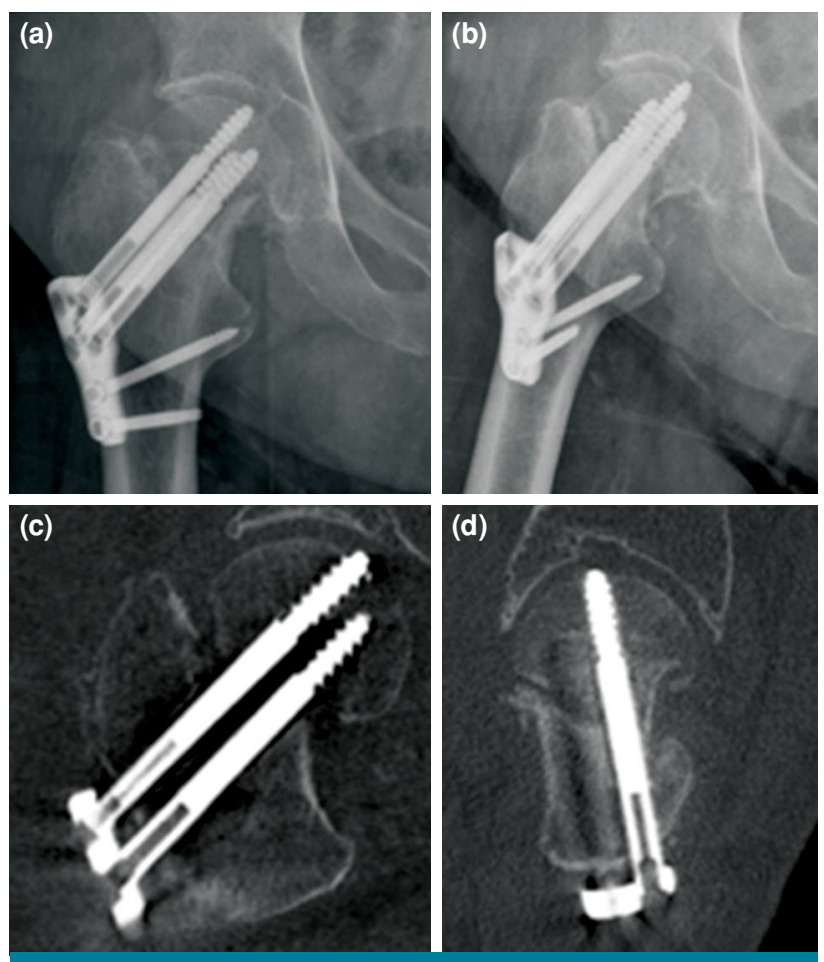

FIGURE 3. (a, b) Eleven-month follow-up hip radiographs and (c, d) multiplanar reconstruction from computer tomography show femoral neck shortening and bony nonunion. (c, d) Inferior screws are completely collapsed and proximal screw is extended $3 \mathrm{~mm}$ and cutting through femoral head.

telescrew sliding capability at some time point due to jamming, as suggested by Eschler et al. ${ }^{[10]}$

In our present case, the situation was different. The cut-through with femoral head perforation occurred in two different telescrews at two time points during the patient's postoperative course, but neither of the telescrews failed to slide. The first perforating telescrew initially slid $3 \mathrm{~mm}$, then extended $5 \mathrm{~mm}$ and then fully collapsed $20 \mathrm{~mm}$ and turned nonperforating. The second telescrew fully telescoped and then slightly extended and perforated the femoral head. These telescrew migrations resemble the reversed Z-effect and the Z-effect, complications found after stabilization of unstable trochanteric fractures with PFNs. ${ }^{[12,14,15]}$ In the Z-effect phenomenon, the inferior lag screw of the PFN construct slides laterally and the superior antirotation screw cuts out from the femoral head. ${ }^{[12]}$ The Z-effect phenomenon was reproduced in laboratory settings by Strauss et al., ${ }^{[16]}$ who observed that cyclical vertical loading led to inferior lag screw lateral sliding if there was greater compressive strength in the femoral head than in the femoral neck. ${ }^{[16]}$ Medial femoral neck element migration was reproduced in various types 
of intramedullary nails by Weil et al., ${ }^{[14]}$ but only with regard to nail toggling within the femoral canal. The exception was a PFN in which even prevention from toggling did not stop medial migration of the distal lag screw. They hypothesized that two-screw devices probably involve a different mechanism of medial screw migration. ${ }^{[14]}$

In our case of osteoporotic displaced FNF treated with DLP, we reported consecutive femoral head perforations with non-jammed and extended telescrews. ${ }^{[17]}$ The development of this failure resembles the reversed Z- and Z-effects found in PFN. We can only speculate as to the explanation for these phenomena: possibly denser bone superiorly than medially in the femoral head, or medial femoral neck fragmentation which can lead to greater instability across the femoral neck with unpredictable telescrew loading during the gait cycle. The real frequency of these phenomena and their proportion related to total cut-through in DPL as well as their risk factors and biomechanical explanation require further studies.

\section{Acknowledgments}

Special thanks to Andrew Billingham for proofreading the manuscript.

\section{Declaration of conflicting interests}

The authors declared no conflicts of interest with respect to the authorship and/or publication of this article.

\section{Funding}

The authors received no financial support for the research and/or authorship of this article.

\section{REFERENCES}

1. Fixation using Alternative Implants for the Treatment of Hip fractures (FAITH) Investigators. Fracture fixation in the operative management of hip fractures (FAITH): an international, multicentre, randomised controlled trial. Lancet 2017;389:1519-27.

2. Şahin A, Agar A, Gülabi D, Ertürk C. Comparison of dynamic hip screw and antirotation screw with cannulated screw in the treatment of transcervical collum femoris fractures. Jt Dis Relat Surg 2020;31:320-7.

3. Weil YA, Qawasmi F, Liebergall M, Mosheiff R, Khoury A. Use of fully threaded cannulated screws decreases femoral neck shortening after fixation of femoral neck fractures. Arch Orthop Trauma Surg 2018;138:661-7.
4. Parker MJ, Stedtfeld HW. Internal fixation of intracapsular hip fractures with a dynamic locking plate: initial experience and results for 83 patients treated with a new implant. Injury 2010;41:348-51.

5. Parker MJ, Raghavan R, Gurusamy K. Incidence of fracturehealing complications after femoral neck fractures. Clin Orthop Relat Res 2007;458:175-9.

6. Yin $\mathrm{H}$, Pan Z, Jiang H. Is dynamic locking plate(Targon FN) a better choice for treating of intracapsular hip fracture? A meta-analysis. Int J Surg 2018;52:30-34.

7. Biber R, Brem M, Bail HJ. Targon Femoral Neck for femoralneck fracture fixation: lessons learnt from a series of one hundred and thirty five consecutive cases. Int Orthop 2014;38:595-9.

8. Xiong WF, Chang SM, Zhang YQ, Hu SJ, Du SC. Inferior calcar buttress reduction pattern for displaced femoral neck fractures in young adults: a preliminary report and an effective alternative. J Orthop Surg Res 2019;14:70.

9. Parker M, Cawley S, Palial V. Internal fixation of intracapsular fractures of the hip using a dynamic locking plate: Two-year follow-up of 320 patients. Bone Joint J 2013;95-B:1402-5.

10. Eschler A, Brandt S, Gierer P, Mittlmeier T, Gradl G. Angular stable multiple screw fixation (Targon FN) versus standard SHS for the fixation of femoral neck fractures. Injury 2014;45 Suppl 1:S76-80.

11. Körver RJ, Wieland AW, Kaarsemaker S, Nieuwenhuis JJ, Janzing HM. Clinical experience, primary results and pitfalls in the treatment of intracapsular hip fractures with the Targon ${ }^{\circledR}$ FN locking plate. Injury 2013;44:1926-9.

12. Werner-Tutschku W, Lajtai G, Schmiedhuber G, Lang T, Pirkl C, Orthner E. Intra- and perioperative complications in the stabilization of per- and subtrochanteric femoral fractures by means of PFN. Unfallchirurg 2002;105:881-5.

13. Singh M, Nagrath AR, Maini PS. Changes in trabecular pattern of the upper end of the femur as an index of osteoporosis. J Bone Joint Surg Am 1970;52:457-67.

14. Weil YA, Gardner MJ, Mikhail G, Pierson G, Helfet DL, Lorich DG. Medial migration of intramedullary hip fixation devices: a biomechanical analysis. Arch Orthop Trauma Surg 2008;128:227-34.

15. Boldin C, Seibert FJ, Fankhauser F, Peicha G, Grechenig W, Szyszkowitz R. The proximal femoral nail (PFN)--a minimal invasive treatment of unstable proximal femoral fractures: a prospective study of 55 patients with a followup of 15 months. Acta Orthop Scand 2003;74:53-8.

16. Strauss EJ, Kummer FJ, Koval KJ, Egol KA. The "Z-effect" phenomenon defined: a laboratory study. J Orthop Res 2007;25:1568-73.

17. Atik OŞ. Is there something new and interesting in my article? Eklem Hastalik Cerrahisi 2019;30:69. 